\title{
A segregation index combining phenotypic (clinical characteristics) and genotypic (gene expression) biomarkers from a urine sample to triage out patients presenting with hematuria who have a low probability of urothelial carcinoma
}

Laimonis Kavalieris ${ }^{1}$, Paul J O'Sullivann ${ }^{1}$, James M Suttie ${ }^{1 *}$, Brent K Pownall ${ }^{2}$, Peter J Gilling ${ }^{3}$, Christophe Chemasle ${ }^{4}$ and David G Darling ${ }^{1}$

\begin{abstract}
Background: Hematuria can be symptomatic of urothelial carcinoma (UC) and ruling out patients with benign causes during primary evaluation is challenging. Patients with hematuria undergoing urological work-ups place significant clinical and financial burdens on healthcare systems. Current clinical evaluation involves processes that individually lack the sensitivity for accurate determination of UC. Algorithms and nomograms combining genotypic and phenotypic variables have largely focused on cancer detection and failed to improve performance. This study aimed to develop and validate a model incorporating both genotypic and phenotypic variables with high sensitivity and a high negative predictive value (NPV) combined to triage out patients with hematuria who have a low probability of having UC and may not require urological work-up.

Methods: Expression of IGFBP5, HOXA13, MDK, CDK1 and CXCR2 genes in a voided urine sample (genotypic) and age, gender, frequency of macrohematuria and smoking history (phenotypic) data were collected from 587 patients with macrohematuria. Logistic regression was used to develop predictive models for UC. A combined genotypic-phenotypic model (G + P INDEX) was compared with genotypic (G INDEX) and phenotypic (P INDEX) models. Area under receiver operating characteristic curves (AUC) defined the performance of each INDEX: high sensitivity, NPV $>0.97$ and a high test-negative rate was considered optimal for triaging out patients. The robustness of the G + P INDEX was tested in 40 microhematuria patients without UC.

Results: The G +P INDEX offered a bias-corrected AUC of 0.86 compared with 0.61 and 0.83 , for the $P$ and $G$ INDEXs respectively. When the test-negative rate was 0.4 , the $G+P$ INDEX (sensitivity $=0.95 ; N P V=0.98$ ) offered improved performance compared with the G INDEX (sensitivity $=0.86$; NPV $=0.96$ ). $80 \%$ of patients with microhematuria who did not have UC were correctly triaged out using the G + P INDEX, therefore not requiring a full urological work-up.

(Continued on next page)
\end{abstract}

\footnotetext{
* Correspondence: jimmy.suttie@pelnz.com

'Pacific Edge Ltd, Dunedin, New Zealand

Full list of author information is available at the end of the article
} 
(Continued from previous page)

Conclusion: The adoption of G + P INDEX enables a significant change in clinical utility. G + P INDEX can be used to segregate hematuria patients with a low probability of UC with a high degree of confidence in the primary evaluation. Triaging out low-probability patients early significantly reduces the need for expensive and invasive work-ups, thereby lowering diagnosis-related adverse events and costs.

Keywords: Macroscopic hematuria, Microscopic hematuria, Urine test, Urothelial carcinoma, Genotypic biomarkers, Gene expression, Phenotypic biomarkers, Triage, Clinical pathway, Urology

\section{Background}

Hematuria, which is most often associated with causes such as benign prostatic enlargement, infection or urinary calculi, but is also symptomatic of urothelial carcinoma (UC), is estimated to occur in between 1 and $22 \%$ of patients in a general population [1,2]. Macroscopic (macro-) hematuria is characterized by a visible color change in the urine of patients, while microscopic (micro-) hematuria is defined more precisely as the presence of $\geq 3$ red blood cells per high-powered field (RBCs/HPF) in three concurrently collected urine samples [2]. The overall prevalence of UC in patients with microhematuria has been reported to be approximately $4 \%$, whereas several studies have consistently shown that the prevalence of UC is much higher in patients with macrohematuria, ranging from approximately $12-23 \%$ [2-6], yet up to four times as many patients with microversus macrohematuria present for urological evaluation [7]. Notably, given that recent changes to the American Urological Association (AUA) guidelines [2] have seen the threshold for asymptomatic microhematuria (AMH) lowered to $\geq 3 \mathrm{RBCs} / \mathrm{HPF}$ in a single sample, and even lower thresholds ( $\geq 1 \mathrm{RBC} / \mathrm{HPF}$ ) have been proposed [8], a consequential increase in the number of patients with hematuria who will undergo a urological work-up to investigate potential UC and a corresponding increase in the overall clinical and financial burden of these patients on healthcare systems is expected.

Such hematuria-related referrals place a significant clinical burden on urologists, as all patients must undergo a full work-up to provide an often inconclusive diagnosis. Furthermore, the existing diagnostic tests - many of which are invasive or have high radiation loadings - can have a detrimental effect on patient quality of life (QoL), especially if the patient receives repeated cystoscopies as mandated in the current guidelines [2]. It has been reported that for cystoscopies performed without prophylactic antibiotics, $22 \%$ of patients had asymptomatic bacteriuria and $1.9 \%$ of patients developed a febrile urinary tract infection (UTI) within 30 days [9]. Other studies have also reported a high prevalence of macrohematuria, pain on voiding and transient erectile dysfunction in men following cystoscopy $[10,11]$.

Healthcare systems also incur a significant financial burden as a result of patients with hematuria undergoing a full urological work up $[12,13]$ and it has been concluded that urine cytology adds costs without offering any significant diagnostic benefit [14-16]. Consequently, integrating an accurate, non-invasive test into the primary clinical work-up of patients presenting with hematuria allows physicians to effectively triage patients with hematuria, thereby reducing the number of patients undergoing a full urological work-up and investigative cystoscopy for UC, and offers significant benefits to both patients and healthcare systems [15-19].

Several clinical prognostic characteristics, including age, gender, smoking history and degree of hematuria, are well-established as risk factors for UC in patients with hematuria [3,20-22]. Recently, several groups have attempted to develop models based on clinical prognostic characteristics to predict the risk of UC in patients with hematuria [20-22], but critically, these models offer limited accuracy and have largely focused on detecting patients with UC rather than ruling out patients who do not have disease. These detection-focused models have therefore been insufficient to reliably identify patients with disease during a primary evaluation, even if used in combination with urine cytology [20-22].

Despite the higher incidence of UC in patients presenting with macrohematuria, a number of studies show there is no significant difference in the distribution of UC by grade and stage in patients presenting with micro- compared with those presenting with macrohematuria [5,23-25]. Therefore, the AUA recommends that all patients with macrohematuria or $\mathrm{AMH}$ be referred to a urologist for a full urological work-up, as severity of hematuria is not sufficiently predictive for the presence of UC [2]. However, as patients with hematuria may only undergo limited urinalysis in a primary evaluation, consisting of cytology and in some cases imaging studies, such as ultrasound, a full urological work-up is often necessary to conclusively detect or rule out UC. While urine cytology is specified in current guidelines and routinely used in patients with suspected UC, cytology results are often inconclusive with atypical or suspicious findings and also suffer from a low diagnostic yield driven by a relatively high risk of false negative results for patients with UC-related hematuria [2,26,27]. Consequently, it can be difficult to rule out benign causes of 
hematuria, whether macrohematuria or $\mathrm{AMH}$, during a primary evaluation, especially if UC-related hematuria is intermittent and appears to resolve following treatment for a benign cause [12].

A number of gene-based studies have set out to profile urinary biomarkers in patients with UC, and these biomarkers may be useful in their own right for detecting disease $[28,29]$. An opportunity also exists to triage out patients on the basis of their gene expression profile and clinical characteristics. Combining NMP22 enzyme-linked immunosorbent assay (ELISA) tests or a panel of gene markers with clinical characteristics has been shown to improve diagnostic accuracy compared with clinical characteristics alone, but these combined models have not yet delivered significant advances in overall diagnostic accuracy, especially when attempting to identify low-risk patients $[30,31]$. Nevertheless it is considered that incorporating clinical factors and specific gene expression into a combined algorithm is likely to provide the best guidance for diagnosing and managing patients with hematuria or UC [32].

Cxbladder Detect (Pacific Edge Ltd., Dunedin, New Zealand), a multigene test performed on unfractionated urine has previously been shown to be more sensitive than urine cytology and NMP22 for detecting UC in patients with macrohematuria [33] and more accurate than urine cytology, NMP22 and fluorescence in situ hybridization (FISH) in a comparative analysis (Breen, Kasabov, O'Sullivan, et al., unpublished observations). Cxbladder Detect uses quantitative reverse transcription polymerase chain reaction (RT-qPCR) technology to quantify five mRNA markers, four markers that are overexpressed in UC alongside a fifth marker that is elevated in non-malignant inflammatory conditions, and offers a high level of specificity and sensitivity when used to detect UC in patients presenting with hematuria [33]. It was hypothesized that an integrated model combining high-performance genetic biomarkers with phenotypic variables collected from the same patients will provide superior clinical resolution using high sensitivity (i.e. a low probability of a patient with UC receiving a false negative result), high negative predictive value (i.e. a high proportion of all negative results being true) and a high testnegative rate to enable the accurate triage of patients who have a low probability of UC. These genotypic and phenotypic variables when combined into a novel segregation model enable patients with hematuria who have a low probability of UC to be identified and triaged, as opposed to undergoing a full urological work-up.

\section{Methods}

\section{Patient selection}

A prospective sample of 695 patients has been analysed, where true clinical outcome was determined using a conventional clinical evaluation. The study sample consisting of an initial cohort of patients with hematuria was consented and sampled as previously described [33], where a consecutive series of 517 patients with a recent history of macrohematuria, aged $\geq 45$ years and without a prior history of $\mathrm{UC}$, were recruited prospectively from nine urology clinics in Australia and New Zealand. These patients were followed for three months for determination of UC status or alternative diagnosis, including upper urinary tract carcinoma [33] following multigene analysis of urine samples, with a positive UC diagnosis being based on cystoscopic appearance and histopathologic examination. The stage of disease was classified according to the TNM staging criteria determined by pathology and diagnostic imaging investigations and tumor grade was classified according to local pathology practice, using the 1998 World Health Organization (WHO)/International Society of Urological Pathology (ISUP) consensus classification [34].

Additional cohorts of 94 and 84 patients undergoing urological investigations following a macrohematuria event were subsequently recruited from two centers in New Zealand between March 2012 and April 2013 and included in the development of models. Centers were selected to participate on the basis of their previous experience participating in the initial study and their willingness to evaluate the Cxbladder Detect product within individual clinical settings.

An additional set of 45 patients presenting with microhematuria were consented and prospectively sampled prior to cystoscopic investigation for possible UC. Samples collected were used for further validation of the $G+P$ INDEX, as set out below.

Eligibility criteria were similar to those of [33], except that patients aged $\geq 18$ years and those who had previously undergone a cystoscopy to investigate UC that proved to be negative were eligible for enrolment. Furthermore, as in [33], patients exhibiting symptoms indicative of a UTI, or bladder or renal calculi, were excluded.

Ethical approvals were obtained from the New Zealand multiregion Health and Disability Ethics Committees, as required. All study participants provided informed consent prior to investigation.

\section{Urine sample collection and assessment}

To provide gene expression data, a single mid-stream urine sample was collected from participants using the Urine Sampling System from Pacific Edge. Multigene analysis of samples from all studies was carried out in accordance with the standard operating procedure, as is used for the commercially available Cxbladder Detect multigenic test. All urine samples $(4.5 \mathrm{~mL})$ from the initial cohort were collected at a clinic prior to cystoscopy and transferred to a stabilization liquid via vacuum 
driven aspiration and sent to Pacific Edge within 48 hours. The samples were then stored at $-80^{\circ} \mathrm{C}$ until required for batch analysis. Samples from the subsequent cohorts were collected in the same manner, but shipped to Pacific Edge at ambient temperature and processed within 7 days of sample collection in accordance with revised quality control (QC) limits and tolerance testing performed at the Pacific Edge diagnostic laboratory.

\section{Definitions}

The term 'phenotypic' has been used to define clinical prognostic characteristics and to distinguish them from gene expression-based biomarkers that have been broadly defined as 'genotypic' variables.

\section{Statistical analysis}

Univariate logistic regression was used to estimate the unadjusted (raw) log odds ratio (logOR) co-efficients for four binary phenotypic variables associated with UC: age, gender, smoking history and average daily frequency of hematuria events observed by the patient during the most recent hematuria episode (Hfreq; see Table 1). For microscopic hematuria, no events were observed, hence Hfreq $=0$. Multivariate logistic regression on all four phenotypic variables was used to generate adjusted $\log$ OR co-efficients in the phenotypic model (P INDEX).

G INDEX was developed using logistic regression to determine the association between UC and mRNA concentrations for the five Cxbladder Detect genes (IGFBP5, HOXA13, MDK, CDK1 and CXCR2) in urine samples. A multivariate genotypic-phenotypic model (G + P INDEX) was generated using a combination of all nine variables from the G INDEX and P INDEX. These linear models determined the $\log \mathrm{OR}$ from which the probability of a patient having UC can be derived.

The relative performance of each of model was illustrated in receiver operating characteristic (ROC) curves plotting the true positive rate versus the false positive rate when testing for $\mathrm{UC}$, as determined by each model. Area under the curve (AUC) was used to summarize the performance of each model with an AUC approaching 1 deemed to be optimal.

To reduce potential bias when model estimation and prediction are performed on the same data set, a

Table 1 Definitions of binary phenotypic variables associated with UC and their corresponding scores

\begin{tabular}{lll}
\hline $\begin{array}{l}\text { Phenotypic } \\
\text { parameter }\end{array}$ & Score & $\mathbf{1}$ \\
\cline { 2 - 3 } & $\mathbf{0}$ & Male \\
\hline Gender & Female & $\geq 60$ years \\
Age & $<60$ years & Current or past smoker \\
Smoking history & Never smoked & $>1$ event/day \\
Hfreq & $\leq 1$ event/day & \\
\hline
\end{tabular}

bias-corrected AUC was calculated for each of the three logistic regression models using bootstrap resampling [35]. The difference between the nominal AUC from the original sample and the average AUC from the bootstrap samples is an estimate of the sample bias and the nominal AUCs were adjusted accordingly. Bootstrap estimates of bias-corrected confidence intervals (CIs) were also obtained [36].

Furthermore, it was a design criteria for this clinical test that the performance characteristics of each model must exceed a threshold NPV of 0.97, with as high a sensitivity as possible with the further caveat of having a high test-negative rate. The test-negative rate is selected to provide a high clinical resolution when triaging out patients presenting with hematuria who have a low probability of having UC. Comparisons were made between the G INDEX, P INDEX and G + P INDEX and the performance of each model was determined in terms of sensitivity and NPV with a sufficiently high testnegative rate to provide an effective tool for triaging out patients with hematuria who have a low probability of $\mathrm{UC}$.

\section{Results}

\section{Sample demographics}

Of the 695 patients with macrohematuria registered across the three cohorts, 23 were deemed to be ineligible and samples from a further 85 patients were excluded after enrolment due to the absence of sufficient data or samples failing to meet QC standards (see Figure 1A). In total, samples from 587 patients were available for modelling comprising 72 UC-positive and 515 UC-negative samples.

Of the 45 samples from patients with microhematuria provided, 40 were suitable for analysis with 5 patients deemed ineligible and excluded from the analysis (see Figure 1B). All 45 patients had received a full urological evaluation and clinical truth was confirmed as UCnegative. Full demographic data from both sample populations is presented in Table 2.

\section{Relationship between phenotypic variables and risk of UC in patients with macrohematuria}

Univariate logistic regression analyses of each of the four binary phenotypic variables indicated that age $\geq 60$ years, male gender, a history of smoking and a high frequency of macrohematuria were all associated with an increased risk of UC (Table 3). Adjusted logOR co-efficients were calculated in the multivariate logistic regression model.

$$
\begin{aligned}
& \text { P INDEX }=-3.78+(0.81 \times \text { Age }+0.46 \times \text { Gender } \\
& +0.78 \times \text { Smoking history }+0.59 \times \text { Hfreq })
\end{aligned}
$$




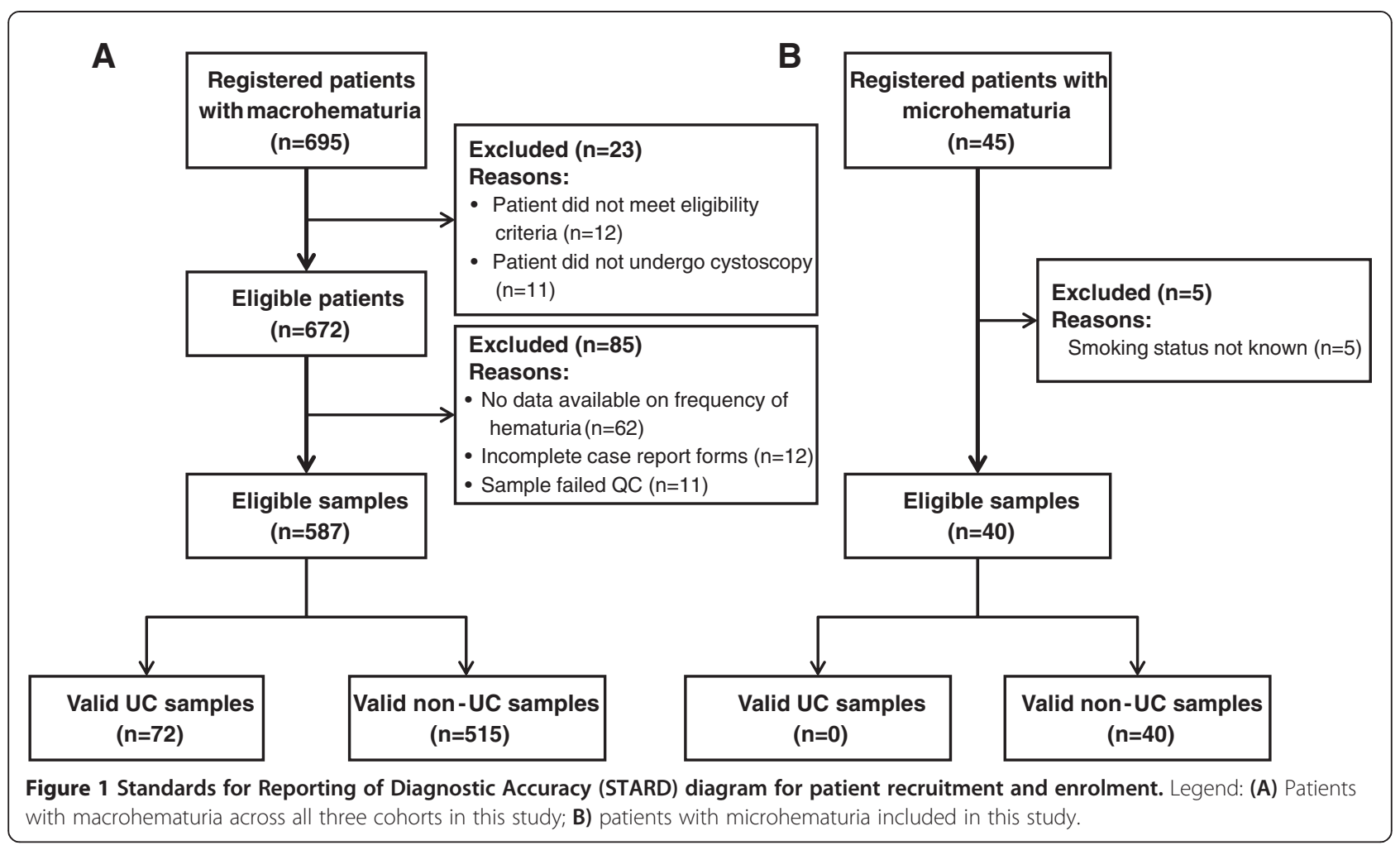

Table 2 Sample population demographics for patients with macro- and microhematuria with complete data

\begin{tabular}{|c|c|c|c|}
\hline Parameter & & Patients with macrohematuria ( $\mathrm{N}=587), \mathrm{n}(\%)$ & Patients with microhematuria $(\mathrm{N}=40), \mathrm{n}(\%)$ \\
\hline \multirow[t]{6}{*}{ Age, years } & $<40$ & $8(1.4)$ & \\
\hline & $40-49$ & $47(8.0)$ & $21(52.5)$ \\
\hline & $50-59$ & $107(18.2)$ & \\
\hline & $60-69$ & $143(24.4)$ & \\
\hline & $70-79$ & $181(30.8)$ & $19(47.5)$ \\
\hline & $80-100$ & $101(17.2)$ & \\
\hline \multirow[t]{2}{*}{ Gender } & Female & $113(19.3)$ & $25(62.5)$ \\
\hline & Male & $474(80.7)$ & $15(37.5)$ \\
\hline \multirow[t]{2}{*}{ Smoking history } & Never smoked & $246(41.9)$ & $25(62.5)$ \\
\hline & Current or past smoker & $341(58.1)$ & $15(37.5)$ \\
\hline \multirow[t]{2}{*}{ Hfreq (events/day) } & $\leq 1$ & $332(56.6)$ & $40(100)$ \\
\hline & $>1$ & $255(43.4)$ & - \\
\hline \multirow[t]{6}{*}{ Tumor stage } & Normal & $515(87.7)$ & $40(100)$ \\
\hline & $\mathrm{T} 1$ & $16(2.7)$ & - \\
\hline & T2 & $11(1.9)$ & - \\
\hline & T3 & $2(0.3)$ & - \\
\hline & $\mathrm{Ta}$ & $40(6.8)$ & - \\
\hline & Tis & $3(0.5)$ & - \\
\hline
\end{tabular}


Table 3 Unadjusted and adjusted ORs for UC by phenotypic and genotypic factors for patients with hematuria

\begin{tabular}{|c|c|c|c|c|c|c|}
\hline \multirow{2}{*}{ Phenotypic variables } & & \multirow[b]{2}{*}{ Control (non-UC) } & \multirow[b]{2}{*}{ UC } & \multirow[t]{2}{*}{$\begin{array}{l}\text { Unadjusted } \\
\text { OR }(95 \% \mathrm{Cl})\end{array}$} & \multirow[t]{2}{*}{$\begin{array}{l}\text { Adjusted P variable } \\
\text { OR }(95 \% \mathrm{Cl})\end{array}$} & \multirow[t]{2}{*}{$\begin{array}{l}\text { Adjusted G + P variable } \\
\text { OR }(95 \% \mathrm{Cl})\end{array}$} \\
\hline & & & & & & \\
\hline \multirow[t]{2}{*}{ Age, years } & $<60$ & 151 & 11 & \multirow{2}{*}{$2.30(1.22-4.73)$} & \multirow{2}{*}{$2.24(1.18-4.65)$} & \multirow{2}{*}{$1.89(0.85-4.64)$} \\
\hline & $\geq 60$ & 364 & 61 & & & \\
\hline \multirow[t]{2}{*}{ Gender } & Female & 105 & 8 & \multirow{2}{*}{$2.05(1.01-4.75)$} & \multirow{2}{*}{$1.58(0.76-3.72)$} & \multirow{2}{*}{$3.03(1.12-9.36)$} \\
\hline & Male & 410 & 64 & & & \\
\hline \multirow[t]{2}{*}{ Smoking history } & Never smoked & 227 & 19 & \multirow{2}{*}{$2.20(1.29-3.91)$} & \multirow{2}{*}{$2.19(1.27-3.92)$} & \multirow{2}{*}{$2.67(1.34-5.64)$} \\
\hline & Current or past smoker & 288 & 53 & & & \\
\hline \multirow[t]{3}{*}{ Hfreq (average events/day) } & $\leq 1$ & 300 & 32 & \multirow{2}{*}{$1.74(1.06-2.88)$} & \multirow{2}{*}{$1.80(1.08-3.00)$} & \multirow{2}{*}{$1.76(0.93-3.35)$} \\
\hline & $>1$ & 215 & 40 & & & \\
\hline & & & & $\begin{array}{l}\text { Unadjusted } \\
\text { OR }(95 \% \mathrm{CI})\end{array}$ & $\begin{array}{l}\text { Adjusted G variable } \\
\text { OR }(95 \% \mathrm{Cl})\end{array}$ & $\begin{array}{l}\text { Adjusted G + P variable } \\
\text { OR }(95 \% \mathrm{Cl})\end{array}$ \\
\hline \multicolumn{7}{|l|}{ Genotypic variables } \\
\hline IGFBP5 & & & & $7.34(4.59-12.33)$ & $2.15(1.03-4.58)$ & $2.21(1.03-4.83)$ \\
\hline HOXA13 & & & & $6.27(3.92-10.34)$ & $0.33(0.13-0.83)$ & $0.20(0.07-0.56)$ \\
\hline MDK & & & & $7.10(4.73-11.10)$ & $4.76(1.74-13.62)$ & $8.14(2.64-26.60)$ \\
\hline CDK1 & & & & $7.80(5.11-12.39)$ & $3.47(1.39-9.13)$ & $2.59(0.98-7.18)$ \\
\hline$C X C R 2$ & & & & $1.69(1.36-2.10)$ & $0.65(0.45-0.92)$ & $0.69(0.47-0.98)$ \\
\hline
\end{tabular}

Adjusted P INDEX, G INDEX and G + P INDEX variable ORs are the exponentiated co-efficients in the P INDEX, G INDEX and G + P INDEX, respectively.

where each phenotypic variable is assigned a binary score of 0 or 1 , as designated in Table 1 , and the confidence intervals for the co-efficients are presented in Table 3. The bias-corrected estimate for AUC for the P INDEX is 0.66 (95\% CI: 0.55-0.67; Figure 2).

\section{Relationship between genotypic variables and risk of UC} in patients with macrohematuria

The G INDEX was estimated by logistic regression using the $\log$ mRNA concentrations of the five genes IGFBP5, HOXA13, MDK, CDK1 and CXCR2 in urine samples to predict UC occurrence.

$$
\begin{aligned}
& \text { G INDEX }=-6.22+(0.77 \times \text { IGFBP5 } \\
& -1.11 \times H O X A 13+1.56 \times M D K \\
& +1.24 \times C D K 1-0.43 \times C X C R 2)
\end{aligned}
$$

The G INDEX gives a bias-corrected AUC of 0.83 (95\% CI: 0.74-0.89; Figure 2).

Relationship between genotypic and phenotypic variables and risk of UC in patients with macrohematuria

The five continuous genotypic variables were then combined with the four binary phenotypic variables to estimate the G + P INDEX using multivariate logistic regression.

$$
\begin{aligned}
& \mathrm{G}+\mathrm{P} \mathrm{INDEX}=-8.46+(0.79 \times I G F B P 5 \\
& -1.60 \times H O X A 13+2.10 \times M D K+0.95 \times C D K 1 \\
& -0.38 \times C X C R 2)+(0.64 \times \text { Age }+1.11 \times \text { Gender } \\
& +0.98 \times \text { Smoking history }+0.56 \times \text { Hfreq })
\end{aligned}
$$

The G + P INDEX gives a bias-corrected AUC of 0.86 (95\% CI: 0.80-0.91).

\section{Comparison between G INDEX and G + P INDEX}

There is overlap between the confidence intervals for the G INDEX and G + P INDEX, so a bootstrap version of a paired test was constructed by determining the difference in AUC for the G INDEX and G + P INDEX for each bootstrap sample. Ten thousand bootstrap samples with a sample size of $n=587$ were generated by random sampling with replacement from the original 587 samples available for analysis. The resulting 95\% CI for the difference between models was $0.01-0.08$. Thus the probability that the true difference between the two AUCs is less than 0.01 is $<0.025$, indicating that there is a high likelihood of the AUC for the G + P INDEX being significantly greater than the AUC for the G INDEX.

\section{NPV and sensitivity of models}

The G + P INDEX generated an NPV $>0.97$ over the range of test-negative rates from 0.2 to 0.7 and was almost always higher than the NPV for the G INDEX model (Figure 3). The G + P INDEX offered performance 


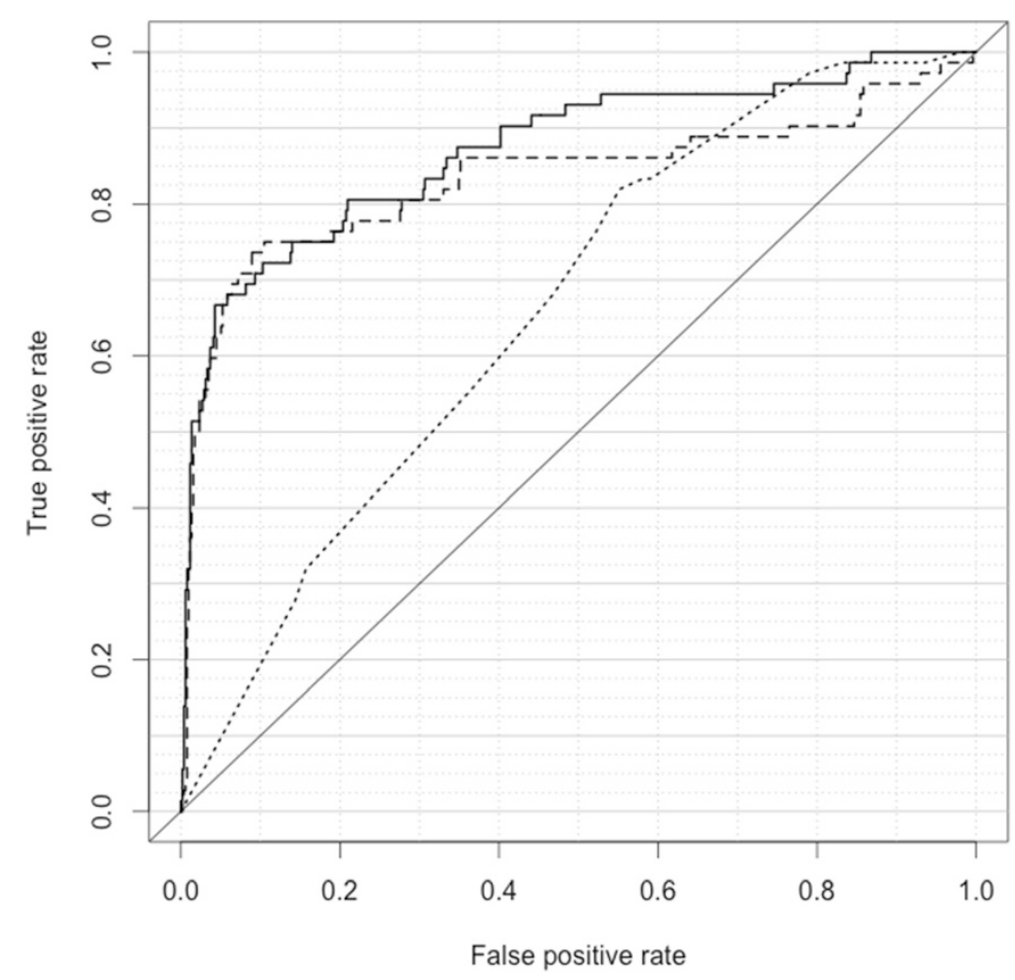

Figure 2 ROC curves representing the three classification models. Legend: P INDEX (dotted), G INDEX (dashed) and G + P INDEX (solid).

characteristics of sensitivity of 0.95 and NPV 0.98 when the test-negative rate was 0.4 (Table 4; Figure 3). In contrast, the G INDEX only achieved sensitivity of 0.86 and an NPV of 0.96 when the test-negative rate was 0.4 (Table 4).

\section{Application of the G + P INDEX in patients with microhematuria}

While the G + P INDEX was developed using data from patients with macrohematuria, its robustness was tested in a further 40 samples from patients with microhematuria (Hfreq $=0$ for all microhematuria patients). A higher test-negative rate was expected in a microhematuria population as the incidence of UC is lower in this population, and using a test-negative rate of $0.4,32$ (80\%) patients tested negative and would be correctly triaged out, therefore not requiring a full urological work-up for the determination of UC.

\section{Discussion}

This study defines a clinical tool that offers clinicians and physicians the ability to effectively triage-out patients presenting with hematuria from the need to have a full urological work-up for the detection of UC. The study presents an internally validated genotypicphenotypic model, G + P INDEX, with bootstrap-based
CI estimates, that offers a combination of high sensitivity and high NPV (i.e. a low probability of an individual patient with UC providing a false-negative result and a high proportion of all negative results being true) that is not offered by models derived exclusively from genotypic or phenotypic data alone. This provides clinicians and physicians with a unique opportunity to triage out patients with both micro- and macrohematuria, in particular by identifying patients with a low risk of having UC who do not require a full urological work-up.

A high test-negative rate in the context of high sensitivity is an important consideration for an effective triage-out test that aims to direct patients with a low probability of UC away from a full clinical work-up [37]. Accordingly, at a test-negative rate of 0.4 the sensitivity of the G + P INDEX presented here maximizes both the sensitivity and NPV (0.95 and 0.98 , respectively). This can be compared with the best fit selected from the genotypic model published in [33] (sensitivity $=0.82$; $\mathrm{NPV}=0.97$ ) and is also comparable with the sensitivity and NPV of both cystoscopy (sensitivity $=0.89-0.98$; $\mathrm{NPV}=0.99)$ and virtual cystoscopy using computed tomography (CT) scans or magnetic resonance imaging (MRI) (sensitivity $=0.94$ and 0.91, respectively) [38-40].

It is acknowledged that the population used to derive the $G$ INDEX, $P$ INDEX and $G+P$ INDEX in this 


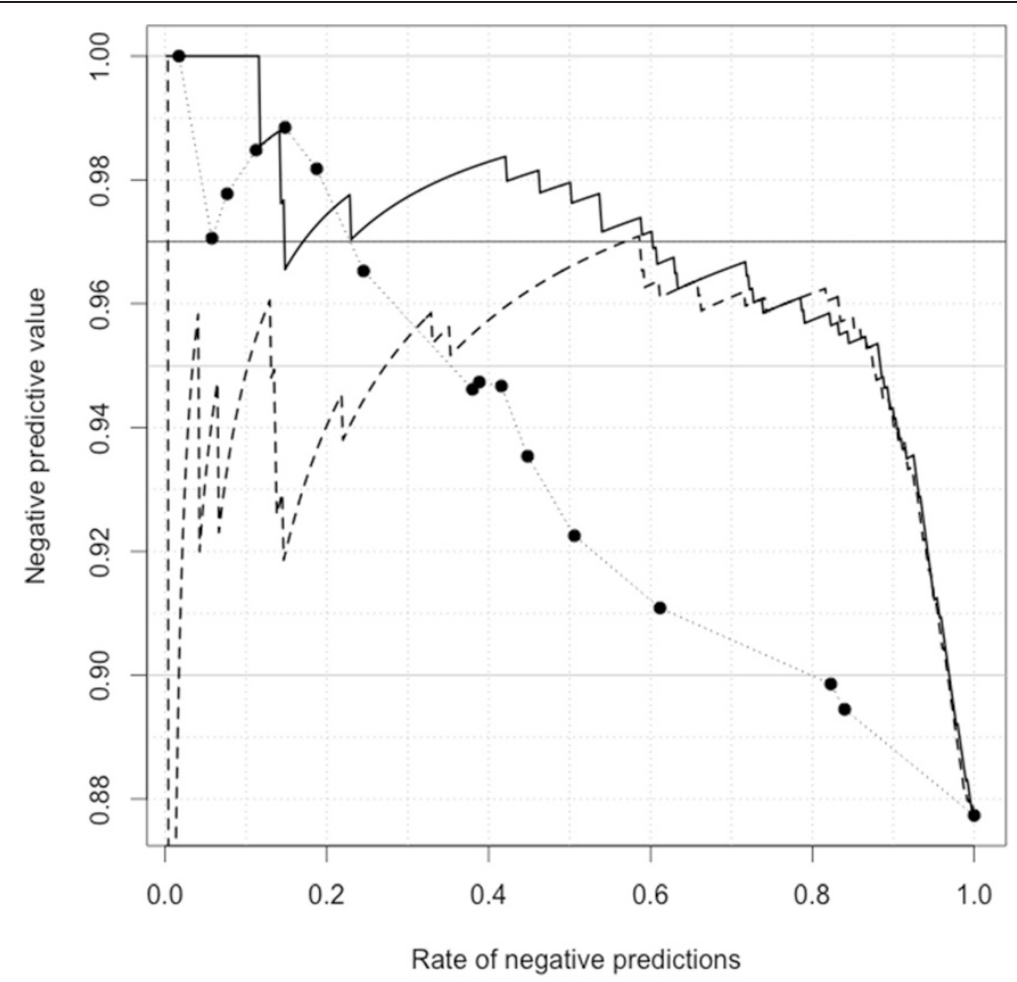

Figure 3 NPV versus proportion of patients with hematuria testing negative according to the three classification models. Legend: P INDEX (dotted), G INDEX (dashed) and G + P INDEX (solid) models. Note that the curve for the P INDEX is discrete as only 16 possible combinations of phenotypic variables are possible. The only possible values taken are indicated by the prominent points on this curve.

instance consisted of patients with macrohematuria. Therefore, the derived NPV values are only applicable to the macrohematuria population. The sensitivity of 0.95 (95\% CI: 0.86-0.98) may, however, be applied across populations. Presuming that patients with and without UC are similarly distributed amongst the micro- and macrohematuria patient populations as depicted in [5,23-25], and that expected UC prevalence is $4 \%$ in the microhematuria population, a higher NPV and testnegative rate can be expected in the target microhematuria population.

By applying the G+P INDEX to the sample population of patients with microhematuria who do not have UC it was shown that $80 \%$ of the patients would have been triaged out on the basis of the result. Only 20\% would be referred for a full urological work-up. This compares with conventional guidelines that would currently see all of the patients (100\%) with microhematuria that cannot be attributed to a benign cause undergoing a full urological work-up, incurring significant unnecessary costs and negatively impacting patient QoL.

Severity of hematuria is correlated with the probability of a patient having UC, but not the stage or grade of any tumor, and an estimated $96 \%$ and $77-88 \%$ of patients with micro- and macrohematuria, respectively, referred to a urologist will not have UC [2-6]. Consequently, avoiding potentially unnecessary urological work-ups for patients with hematuria has several benefits. Cystoscopy may be associated with adverse effects, such as pain on voiding, bleeding, UTIs, male sexual dysfunction and the anxiety that accompanies an inconclusive or unconfirmed UC diagnosis [9-11]. Most notably, this novel approach has the potential to reduce the burden on resources and the financial cost associated with a full urological work-up on UC-negative patients. For example, in the UK, avoiding cystoscopy in patients with hematuria with an initial negative cytology and/or tumor biomarker test has been estimated to save approximately US\$770 per patient ( $£ 483$ per patient) evaluated [13]. The $G+P$ INDEX described here provides an effective alternative to the use of urine cytology when used in a primary evaluation setting. This is particularly relevant in settings where primary evaluation is carried out by primary care physicians.

On this basis, if we assign an arbitrary 'nominal cost' of US $\$ 4,500$ for each full urological work up, the total cost for working up 1,000 patients with microhematuria would approach US $\$ 4.5$ million. In contrast, if $80 \%$ of patients with microhematuria are triaged out using the $\mathrm{G}+\mathrm{P}$ INDEX at an arbitrary nominal cost of US\$2,500, 
Table 4 Performance characteristics of each model when thresholds are set for varying test negative rates as determined on the macroscopic hematuria population

\begin{tabular}{|c|c|c|c|c|}
\hline Threshold (logOR) & Test-negative rate $(95 \% \mathrm{Cl})$ & NPV $(95 \% \mathrm{Cl})$ & Sensitivity $(95 \% \mathrm{Cl})$ & Specificity $(95 \% \mathrm{Cl})$ \\
\hline \multicolumn{5}{|l|}{ P INDEX } \\
\hline-2.54 & $0.25(0.21-0.28)$ & $0.97(0.92-0.99)$ & $0.93(0.85-0.98)$ & $0.27(0.23-0.31)$ \\
\hline-2.52 & $0.38(0.34-0.42)$ & $0.95(0.91-0.97)$ & $0.83(0.74-0.91)$ & $0.41(0.37-0.45)$ \\
\hline-2.39 & $0.42(0.37-0.45)$ & $0.95(0.91-0.97)$ & $0.82(0.72-0.90)$ & $0.45(0.40-0.49)$ \\
\hline-1.95 & $0.51(0.47-0.54)$ & $0.92(0.89-0.95)$ & $0.68(0.56-0.78)$ & $0.53(0.49-0.57)$ \\
\hline-1.93 & $0.51(0.46-0.55)$ & $0.92(0.89-0.95)$ & $0.68(0.56-0.78)$ & $0.53(0.49-0.58)$ \\
\hline-1.73 & $0.82(0.79-0.85)$ & $0.90(0.87-0.92)$ & $0.32(0.22-0.43)$ & $0.84(0.81-0.87)$ \\
\hline \multicolumn{5}{|l|}{ G INDEX } \\
\hline-3.46 & $0.20(0.17-0.23)$ & $0.94(0.88-0.97)$ & $0.90(0.80-0.95)$ & $0.22(0.18-0.25)$ \\
\hline-3.23 & $0.30(0.26-0.34)$ & $0.95(0.91-0.98)$ & $0.89(0.80-0.95)$ & $0.33(0.28-0.37)$ \\
\hline-3.04 & $0.40(0.36-0.44)$ & $0.96(0.92-0.98)$ & $0.86(0.77-0.93)$ & $0.44(0.40-0.48)$ \\
\hline-2.86 & $0.50(0.46-0.54)$ & $0.97(0.94-0.98)$ & $0.86(0.77-0.93)$ & $0.55(0.51-0.59)$ \\
\hline-2.63 & $0.60(0.56-0.63)$ & $0.96(0.94-0.98)$ & $0.82(0.71-0.90)$ & $0.66(0.62-0.69)$ \\
\hline-2.41 & $0.70(0.66-0.73)$ & $0.96(0.94-0.98)$ & $0.78(0.65-0.86)$ & $0.77(0.73-0.80)$ \\
\hline \multicolumn{5}{|l|}{$\mathrm{G}+\mathrm{P}$ INDEX } \\
\hline-4.02 & $0.20(0.17-0.23)$ & $0.97(0.93-0.99)$ & $0.96(0.88-0.99)$ & $0.22(0.19-0.26)$ \\
\hline-3.67 & $0.30(0.26-0.33)$ & $0.98(0.94-0.99)$ & $0.94(0.87-0.99)$ & $0.33(0.29-0.37)$ \\
\hline-3.33 & $0.40(0.36-0.44)$ & $0.98(0.95-1.00)$ & $0.95(0.86-0.98)$ & $0.45(0.40-0.49)$ \\
\hline-2.99 & $0.50(0.46-0.54)$ & $0.98(0.96-0.99)$ & $0.92(0.83-0.97)$ & $0.56(0.52-0.60)$ \\
\hline-2.71 & $0.60(0.56-0.64)$ & $0.97(0.95-0.99)$ & $0.86(0.76-0.93)$ & $0.67(0.63-0.71)$ \\
\hline-2.37 & $0.70(0.66-0.73)$ & $0.97(0.94-0.98)$ & $0.80(0.70-0.88)$ & $0.77(0.73-0.80)$ \\
\hline
\end{tabular}

the total direct cost of testing and full urological workups for the remaining $20 \%$ of patients would total US $\$ 3.4$ million. This provides a notional net saving in direct costs of approximately US\$1.1 million per 1,000 patients with microhematuria.

The G+P INDEX and G INDEX, developed in this study, use the same genotypic biomarkers used in the genotypic model described in O'Sullivan et al. [33]. However, the G + P INDEX adds a further four phenotypic variables to enhance the ability to segregate patients who have a low probability of UC. The G+P INDEX uses a combinatorial method with a high sensitivity and a high NPV. By contrast, the genotypic model described in O'Sullivan et al. [33] optimizes the balance between sensitivity and specificity to calibrate the model calibrated for the optimal primary detection of UC in symptomatic patients (i.e. presenting with hematuria) who were undergoing a full urological work-up for suspected UC. The G + P INDEX has a significantly different clinical endpoint as no attempt is made to define or select patients with UC. Instead the aim is to confidently rule out those who do not have UC, and as such, all patients not segregated out would progress for a full urological work-up.
While several studies have previously sought to develop predictive models that consider phenotype when assessing the risk of UC in patients presenting with hematuria, the accuracy of phenotype-dependent models alone appears to be limited. For example, Loo et al. [21] prospectively investigated whether phenotypic parameters could be used to identify patients with microhematuria who may not have required a urological referral and full work-up and concluded that age, male gender and a recent diagnosis of macrohematuria were significant predictors of UC. A history of smoking and $>25$ $\mathrm{RBCs} / \mathrm{HPF}$ in a recent urinalysis were not statistically significant predictors of UC, in isolation, but even when included in their 'Hematuria Risk Index' to improve predictive accuracy, this index resulted in an AUC of 0.809 [21]. Interestingly, the phenotypic ORs in this study and those identified by Loo et al. are comparable, with overlapping 95\% CIs for smoking history and gender, and while age, gender and smoking history have similar weightings in each model, the influence of the genotypic component of the G + P INDEX presented here is likely to account for the higher AUC [21].

Likewise, Cha et al. [20] reported that age, smoking history and degree of hematuria, but not gender, were 
significantly correlated with the presence of UC in patients with asymptomatic hematuria and used a multivariate model to develop a nomogram comprised of phenotypic and urine cytology data for predicting UC. As with Loo et al. [21], the reported phenotypic ORs are comparable to those reported here, but even after incorporating urine cytology into the nomogram, the AUC of 0.831 reported in [20] was lower than that of the $G+P$ INDEX.

In another study, Tan et al. [22] retrospectively stratified patients with hematuria who had been referred to a specialist urology clinic into high- and low-risk groups using a nomogram derived from patient age, gender, smoking history and the degree of hematuria. While comparisons with this study must be made with caution given the high proportion of patients who were excluded due to an absence of data ( 80 out of 405 patients), the AUC of 0.804 , sensitivity of 0.900 and NPV of 0.953 were all lower than the G + P INDEX described here.

Several attempts have also been made to improve the accuracy of phenotypic models by supplementing them with the results of urinary biomarker tests. When the nuclear matrix protein NMP22 point of care proteomic assay is used in isolation to detect $\mathrm{UC}$ it has a sensitivity of 0.557 and NPV of 0.968 [17]. Lotan et al. [41] published a multivariable algorithm comprising phenotypic factors, NMP22 and urine cytology with an AUC for predicting UC of 0.826 that was then prospectively validated with an AUC of 0.802 [31]. However, it is important to note that this model attempted to discriminate between high-risk patients who did and did not have $\mathrm{UC}$, as opposed to maximizing sensitivity and NPV to triage-out patients with a low probability of UC.

The improved accuracy obtained with algorithms comprising both genotypic and phenotypic data have previously been demonstrated in breast cancer, in particular [42-45]. Likewise, Mitra et al. [30] used a combination of molecular markers and smoking intensity to calculate a multivariate model that was superior to routine clinicopathological parameters in predicting survival in patients with UC. However, the present study is the first to demonstrate that phenotypic risk factors can be combined with genotypic data to increase the accuracy of a model for separating patients with hematuria into categories requiring differential levels of urological follow up and clinical care rather than survivorship prediction.

When phenotypic data are combined with genotypic data in a model, the resolution of data is likely to impact the accuracy of the model. For example, smoking is a well understood risk factor for UC and is included in most phenotypic models for detecting UC. In Cha et al. [20], Tan et al. [22], Lotan et al. [31,41] and the current study, the binary discriminants never smoked and current/ex-smoker were used, whereas Mitra et al. [30] calculated smoking intensity on the basis of years of smoking and number of cigarettes smoked each day and Loo et al. [21] categorized smokers into never smoked, passive smokers, smokers who had ceased and current smokers. While it is known that the risk of UC increases substantially with exposure to smoking [46], arbitrarily defining phenotypic variables may limit the overall accuracy and utility of phenotypic models. In contrast, an interaction between a patient's genotypic and phenotypic variables would not be unexpected. However, combining the impact of phenotypic factors and genotypic variables in a single tool improved the accuracy of the model described in this study. A similar principle also applies to describing hematuria phenotype. Patients presenting with micro- or macrohematuria are essentially on a biological continuum and have different likelihoods of having UC [2-6,21]. Accordingly, despite all patients with microhematuria in this study having a Hfreq score of 0 , the severity of their hematuria, in combination with other phenotypic factors, is likely to be indirectly accounted for in the genotypic component of the $\mathrm{G}+\mathrm{P}$ INDEX.

\section{Conclusions}

In conclusion, the G $+\mathrm{P}$ INDEX reported here shows a significant opportunity to change clinical utility. $G+P$ INDEX is able to accurately triage out patients who present to their clinician or physician with hematuria, who have a low probability of UC with a high overall test-negative rate, high level of sensitivity and high NPV. This model could be suitable for use by physicians to triage out patients who do not require a full urological work-up, thereby reducing the number of patients with hematuria requiring a full urological evaluation for UC, helping to maintain patient QoL and helping to reduce diagnosis-related costs.

\section{Abbreviations}

AMH: Asymptomatic microhematuria; AUA: American Urological Association; AUC: Area under the curve; $\mathrm{Cl}$ : Confidence interval; CT: Computed tomography; ELISA: Enzyme-linked immunosorbent assay; FISH: Fluorescence in situ hybridization; HPF: High-powered field: logOR: log odds ratio; Hfreq: average daily frequency of hematuria events during the most recent hematuria episode; ISUP: International Society of Urological Pathology; MRI: Magnetic resonance imaging; NPV: Negative predictive value; OR: Odds ratio; QC: Quality control; QoL: Quality of life; RBC: Red blood cell; ROC: Receiver operating characteristic; RT-qPCR: Reverse transcription quantitative polymerase chain reaction; STARD: Standards for Reporting of Diagnostic Accuracy; UC: Urothelial carcinoma; WHO: World Health Organization.

\section{Competing interests}

LK, POS, JS and DD are employees of Pacific Edge Ltd and BP is an employee of Pacific Edge Diagnostics New Zealand Ltd. JS, BP and DD also hold shares and/or share options in Pacific Edge Ltd., a public company whose shares trade on the New Zealand Stock Exchange. DD and POS are listed as applicants in a Patent Cooperation Treaty application, and a corresponding US patent application, covering this technology and JS has advised on the filing of this application. PG is an investigator in clinical trials funded by Pacific Edge Ltd. CC declares that he has no competing interests. 


\section{Authors' contributions}

LK was principally responsible for the statistical analysis and model development and interpreting the data. POS contributed to study management, data collection and interpretation and analysing the published literature. JS contributed to study management and data interpretation and analysing the published literature. BP contributed to the study design and conception, data interpretation and analysis of the published literature. PG contributed to study conception and design, conducted aspects of the study relating to collection of data from patients with macrohematuria and assisted with interpreting the data. CC collected data from patients with microhematuria. DD contributed to the study design and conception, conducting and managing the study, performing statistical analysis, interpreting data and analysing published literature. All authors contributed to the preparation of this manuscript and approved the final draft prior to submission.

\section{Acknowledgements}

The authors thank Dr Mark Dalphin of Pacific Edge Ltd. for his assistance in performing this study and providing editorial suggestions, and Dr Satish Kumar from Plant and Food Research (Hastings, New Zealand) for contributing to the analysis of the combination of G INDEX, P INDEX and G + P INDEX. The authors also thank Blair Hesp of Kainic Medical Communications Ltd. who provided medical writing services on behalf of Pacific Edge Ltd.

\section{Author details}

${ }^{1}$ Pacific Edge Ltd, Dunedin, New Zealand. ${ }^{2}$ Pacific Edge Diagnostics Ltd, Dunedin, New Zealand. ${ }^{3}$ Tauranga Urology Research, Tauranga, New Zealand. ${ }^{4}$ Department of Urology, Palmerston North Hospital, Palmerston North, New Zealand.

Received: 25 November 2014 Accepted: 16 March 2015 Published online: 27 March 2015

\section{References}

1. Kelly JD, Fawcett DP, Goldberg LC. Assessment and management of nonvisible haematuria in primary care. BMJ. 2009;338:a3021.

2. Davis R, Jones JS, Barocas DA, Castle EP, Lang EK, Leveillee RJ, et al. Diagnosis, evaluation and follow-up of asymptomatic microhematuria (AMH) in adults: AUA guideline. J Urol. 2012;188(6 Suppl):2473-81.

3. Sutton JM. Evaluation of hematuria in adults. JAMA. 1990;263:2475-80.

4. Khadra MH, Pickard RS, Charlton M, Powell PH, Neal DE. A prospective analysis of 1,930 patients with hematuria to evaluate clinical practice. J Urol. 2000;163:524-7.

5. Davidson P. Re-design of a haematuria clinic: Assessment of 2346 haematuria patients. J Urol. 2011;185(4S):e495.

6. Price SJ, Shephard EA, Stapley SA, Barraclough K, Hamilton WT. Non-visible versus visible haematuria and bladder cancer risk: A study of electronic records in primary care. Br J Gen Pract. 2014;64:e584-9.

7. Buteau A, Seideman CA, Svatek RS, Youssef RF, Chakrabati G, Reed G, et al. What is evaluation of hematuria by primary care physicians? Use of electronic medical records to assess practice patterns with intermediate follow-up. Urol Oncol. 2014;32:128-34.

8. Jimbo M. Evaluation and management of hematuria. Prim Care. 2010;373:461-72.

9. Herr HW. The risk of urinary tract infection after flexible cystoscopy in bladder tumor patients who did not receive prophylactic antibiotics. J Urol. 2015:193:548-51

10. Burke DM, Shackley DC, O'Reilly PH. The community-based morbidity of flexible cystoscopy. BJU Int. 2002;89:347-9.

11. Stav K, Leibovici D, Goren E, Livshitz A, Siegel YI, Lindner A, et al. Adverse effects of cystoscopy and its impact on patients' quality of life and sexual performance. Isr Med Assoc J. 2004;6:474-8.

12. Rao PK, Jones JS. How to evaluate 'dipstick hematuria': What to do before you refer. Cleve Clin J Med. 2008;75:227-33.

13. Rodgers M, Nixon J, Hempel S, Aho T, Kelly J, Neal D, et al. Diagnostic tests and algorithms used in the investigation of haematuria: systematic reviews and economic evaluation. Health Technol Assess. 2006;10:iii-iv. xi-259.

14. Falebita OA, Lee G, Sweeney P. Urine cytology in the evaluation of urological malignancy revisited: is it still necessary? Urol Int. 2010;84:45-9.
15. Feifer AH, Steinberg J, Tanguay S, Aprikian AG, Brimo F, Kassouf W. Utility of urine cytology in the workup of asymptomatic microscopic hematuria in low-risk patients. Urology. 2010;75:1278-82.

16. Svatek RS, Hollenbeck BK, Holmäng S, Lee R, Kim S, Stenzl A, et al. The economics of bladder cancer: Costs and considerations of caring for this disease. Eur Urol. 2014:66:253-62.

17. Grossman HB, Messing E, Soloway M, Tomera K, Katz G, Berger Y, et al. Detection of bladder cancer using a point-of-care proteomic assay. JAMA. 2005;293:810-6.

18. Friedlander DF, Resnick MJ, You C, Bassett J, Yarlagadda V, Penson DF, et al. Variation in the intensity of hematuria evaluation: a target for primary care quality improvement. Am J Med. 2014;127:633-40.

19. Shinagare $A B$, Silverman $S G$, Gershanik EF, Chang SL, Khorasani R. Evaluating hematuria: impact of guideline adherence on urologic cancer diagnosis. Am J Med. 2014;127:625-32.

20. Cha EK, Tirsar LA, Schwentner C, Hennenlotter J, Christos PJ, Stenzl A, et al. Accurate risk assessment of patients with asymptomatic hematuria for the presence of bladder cancer. World J Urol. 2012;30:847-52

21. Loo RK, Lieberman SF, Slezak JM, Landa LM, Mariani AJ, Nicolaisen G, et al. Stratifying risk of urinary tract malignant tumors in patients with asymptomatic microscopic hematuria. Mayo Clin Proc. 2013;88:129-38.

22. Tan GH, Shah SA, Ann HS, Hemdan SN, Shen LC, Galib NAFA, et al. Stratifying patients with haematuria into high or low risk groups for bladder cancer: a novel clinical scoring system. Asian Pac J Cancer Prev. 2013;14:6327-30.

23. Sultana S, Goodman C, Bryne D, Baxby K. Microscopic haematuria: urological investigation using a standard protocol. Br J Urol. 1996;78:691-8.

24. Sugimura K, Ikemoto S-I, Kawashima H, Nishisaka N, Kishimoto T. Microscopic hematuria as a screening marker for urinary tract malignancies. Int J Urol. 2001:8:1-5.

25. Viswanath S, Zelhof B, Ho E, Sethia K, Mills R. Is routine urine cytology useful in the haematuria clinic? Ann R Coll Surg Engl. 2008:90:153-5.

26. Steiner H, Bergmeister M, Verdorfer I, Granig T, Mikuz G, Bartsch G, et al. Early results of bladder-cancer screening in a high-risk population of heavy smokers. BJU Int. 2008;102:291-6.

27. Yeung $\mathrm{C}$, Dinh $\mathrm{T}$, Lee J. The health economics of bladder cancer: An updated review of the published literature. Pharmacoeconomics. 2014:32:1093-104.

28. Holyoake A, O'Sullivan P, Pollock R, Best T, Watanabe J, Kajita Y, et al. Development of a multiplex RNA urine test for the detection and stratification of transitional cell carcinoma of the bladder. Clin Cancer Res. 2008:14:742-9.

29. Sapre N, Anderson PD, Costello AJ, Hovens CM, Corcoran NM. Gene-based urinary biomarkers for bladder cancer: an unfulfilled promise? Urol Oncol. 2014:32:48.e9-17.

30. Mitra AP, Castelao JE, Hawes D, Tsao-Wei DD, Jiang X, Shi SR, et al. Combination of molecular alterations and smoking intensity predicts bladder cancer outcome. Cancer. 2013;119:756-65

31. Lotan Y, Svatek RS, Krabbe LM, Xylinas E, Klatte T, Shariat SF. Prospective external validation of model for bladder cancer detection. J Urol. 2014:192:1343-8

32. Abogunrin F, O'Kane HF, Ruddock MW, Stevenson M, Reid CN, O'Sullivan

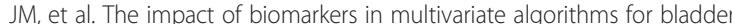
cancer diagnosis in patients with hematuria. Cancer. 2012;118:2641-50.

33. O'Sullivan P, Sharples $K$, Dalphin M, Davidson P, Gilling P, Cambridge L, et al A multigene urine test for the detection and stratification of bladder cancer in patients presenting with hematuria. J Urol. 2012;188:741-7.

34. Epstein Jl, Amin MB, Reuter VR, Mostofi FK. The World Health Organization/ International Society of Urological Pathology consensus classification of urothelial (transitional cell) neoplasms of the urinary bladder. Bladder Consensus Conference Committee. Am J Surg Pathol. 1998;22:1435-48.

35. Efron B, Tibshirani RJ. An introduction to the bootstrap. New York: Chapman Hill; 1993.

36. DiCiccio TJ, Efron B. Bootstrap confidence intervals. Statist Sci. 1996:11:189-228.

37. Van't Hoog AH, Cobelens F, Vassall A, van Kampen S, Dorman SE, Alland D, et al. Optimal triage test characteristics to improve the cost-effectiveness of the Xpert MTB/RIF assay for TB diagnosis: A decision analysis. PLoS One. 2013:8:e82786.

38. Qu X, Huang X, Wu L, Huang G, Ping X, Yan W. Comparison of virtual cystoscopy and ultrasonography for bladder cancer detection: A meta-analysis. Eur J Radiol. 2010;80:188-97. 
39. Mowatt G, N'Dow J, Vale L, Nabi G, Boachie C, Cook JA, et al. Photodynamic diagnosis of bladder cancer compared with white light cystoscopy: Systematic review and meta-analysis. Int J Technol Assess Health Care. 2011;27:3-10.

40. Blick CG, Nazir SA, Mallett S, Turney BW, Onwu NN, Roberts IS, et al. Evaluation of diagnostic strategies for bladder cancer using computed tomography $(\mathrm{CT})$ urography, flexible cystoscopy and voided urine cytology: Results for 778 patients from a hospital haematuria clinic. BJU Int. 2012;110:84-94.

41. Lotan Y, Capitanio U, Shariat SF, Hutterer GC, Karakiewicz PI. Impact of clinical factors, including a point-of-care nuclear matrix protein-22 assay and cytology, on bladder cancer detection. BJU Int. 2009;103:1368-74.

42. Tyrer J, Duffy SW, Cuzick J. A breast cancer prediction model incorporating familial and personal risk factors. Stat Med. 2004;23:1111-30.

43. Dubsky P, Brase JC, Jakesz R, Rudas M, Singer CF, Greil R, et al. The EndoPredict score provides prognostic information on late distant metastases in ER+/HER2- breast cancer patients. Br J Cancer. 2013;109:2959-64

44. Brentnall AR, Evans DG, Cuzick J. Distribution of breast cancer risk from SNPs and classical risk factors in women of routine screening age in the $\mathrm{UK} . \mathrm{Br} J$ Cancer. 2014;110:827-8.

45. Filipits M, Nielsen TO, Rudas M, Greil R, Stöger $H$, Jakesz R, et al. The PAM50 risk-of-recurrence score predicts risk for late distant recurrence after endocrine therapy in postmenopausal women with endocrine-responsive early breast cancer. Clin Cancer Res. 2014;20:1298-305.

46. Krabbe LM, Svatek RS, Shariat SF, Messing E, Lotan Y. Bladder cancer risk: Use of the PLCO and NLST to identify a suitable screening cohort. Urol Oncol. 2015;33:65.e19-25.

\section{Submit your next manuscript to BioMed Central and take full advantage of:}

- Convenient online submission

- Thorough peer review

- No space constraints or color figure charges

- Immediate publication on acceptance

- Inclusion in PubMed, CAS, Scopus and Google Scholar

- Research which is freely available for redistribution 